\title{
Minimally invasive dual iliac screw, dual rod fixation in a rare case of pathological sacral fracture from a paraganglionoma: a technique description
}

\author{
Muhammed Yaser Hasan, MBBS, MRCS(Edin), and Gabriel Liu, MBBCh, MSc, FRCS, FAMS(Orth) \\ University Orthopaedics, Hand and Reconstructive Microsurgery Cluster, National University Health System, National University \\ Hospital, Singapore
}

\begin{abstract}
The management of lumbosacral neoplastic disease can be demanding, often requiring complex reconstruction. In the context of extensive sacral involvement, the risk of iliac screw breakage is greater. Few studies advocate the use of dual iliac screw techniques to reduce implant failure. In this report, the authors have described the first case of percutaneous dual iliac screw, dual rod insertion as part of a minimally invasive spinopelvic stabilization in a patient with a sacral fracture from a paraganglionoma. The patient underwent percutaneous $L-2$ to ilium fixation. A dual iliac screw, dual rod construct was used for stabilizing the left lumbopelvic junction. At the 1-year follow-up, the patient remained asymptomatic, with radiographs showing no signs of instrumentation failure. Minimally invasive dual iliac screw, dual rod fixation is a viable option in cases in which additional stability is required due to extensive neoplastic disease or active individuals have increased functional demands. Short-term results in this report are encouraging; however, more research is warranted to establish the procedure's long-term safety.
\end{abstract}

https://thejns.org/doi/abs/10.3171/2017.3.SPINE161293

KEY WORDS pelvic fixation; iliac bolts; neuroendocrine tumors; sacral tumor; surgical technique

$\mathrm{P}$ ARAGANGLIOMAS are rare, neural crest cell tumors arising from extraadrenal autonomic ganglia. Most are benign, but approximately $20 \%$ possess metastatic potential. ${ }^{15}$ Metastatic paraganglionoma causing sacral fracture and impending cauda equina syndrome is a rare but potentially debilitating condition requiring complex treatment.

Surgical management of a lumbosacral junction (LSJ) metastatic deformity is a unique challenge. The presence of weak pathological bone in a region of high sacroiliac joint stress can undermine any construct and heighten failure risk. ${ }^{4,14}$ Lumbosacral junction fixation techniques over the last 5 decades have evolved from the early use of Harrington rods ${ }^{9}$ to Allen and Ferguson's Galveston technique ${ }^{2}$ to present-generation iliac screw constructs. In 2008 Wang et al. introduced minimally invasive (MI) iliac screw fixation in degenerative spinal disease to reduce wound complications and blood loss..$^{18}$ In a recent study, Liu et al. demonstrated the effective use of MI iliac screw lumbosacral fixation as a palliative treatment in advanced, unstable, LSJ metastatic spinal deformity. ${ }^{11}$ However, with extensive LSJ neoplastic involvement, the risk of iliac screw failure is higher. To provide additional stability and reduce failure risk, open dual iliac screw techniques have been used by a few authors. ${ }^{1,5,19,20}$

To the best of our knowledge, we here report the first instance of MI dual iliac screw, dual rod lumbosacral fixation and MI decompression for the treatment of lumbosacral instability and nerve compression from metastatic paraganglionoma.

\section{Case Report}

History and Examination

A 27-year-old man presented with acute back pain and S-1 radiculopathy. He also reported increasing difficulty walking and passing urine as well as right big toe flexion and extension weakness. Radiographs and CT scan showed 

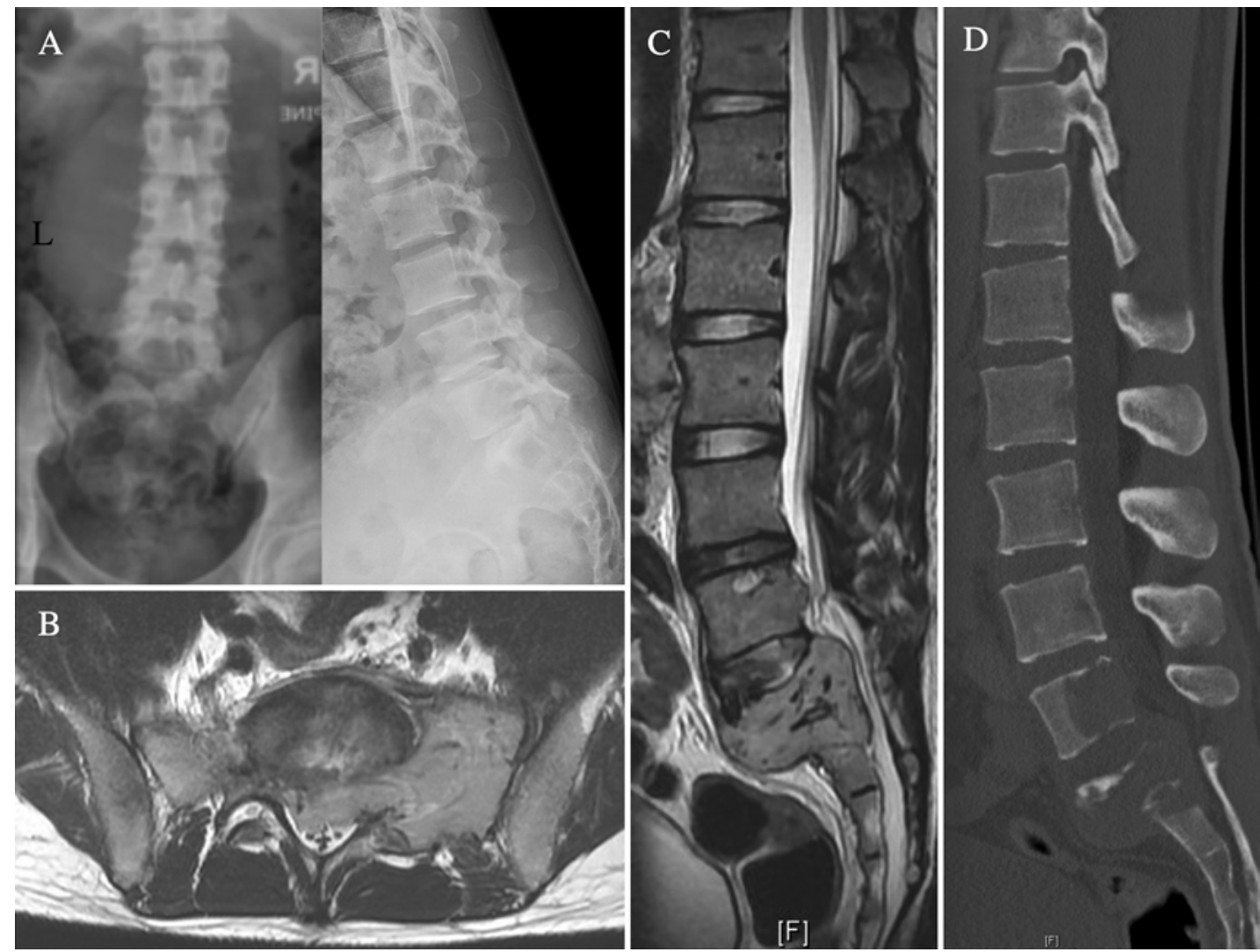

FIG. 1. Left-right anteroposterior (A, left) and lateral (A, right) radiographs and sagittal CT scan (D) showing LSJ destruction. Magnetic resonance images $(\mathbf{B}$, axial; $\mathbf{C}$, lateral) showing extensive tumor infiltration of $\mathbf{S}-1$ with sacral nerve root compression.

a lytic focus from L-5 to the sacrum (Fig. 1A and D). Magnetic resonance imaging showed a large, hypervascular, left retroperitoneal lesion with extensive bony metastasis worse at L-5 and the sacrum with a pathological S-1 fracture (Fig. 1B and C). Abdominal imaging demonstrated a homogeneous left retroperitoneal mass displacing the abdominal vasculature (Fig. 2). A CT-guided biopsy showed the sacral lesion to be a metastatic paraganglionoma. A multidisciplinary team of endocrinologists, general sur-

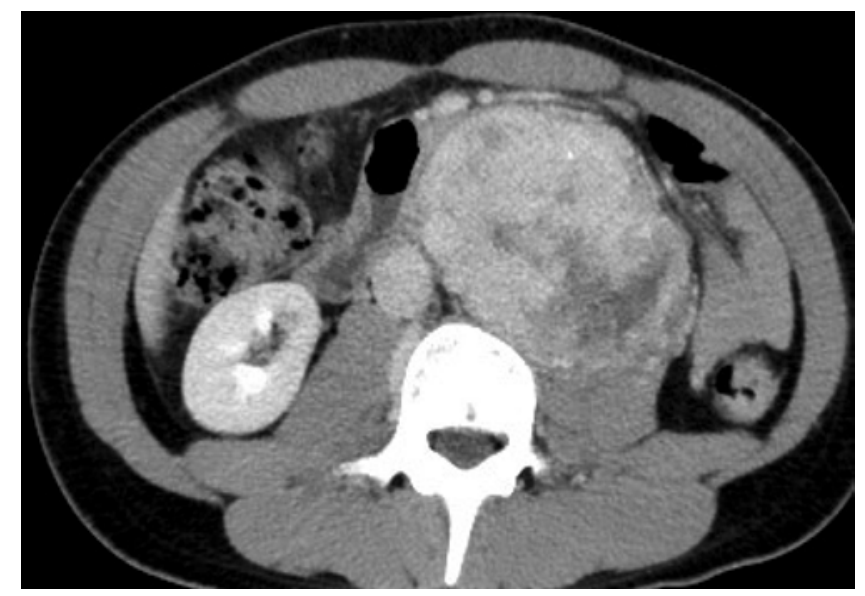

FIG. 2. Abdominal axial CT scan showing a large left retroperitoneal mass displacing the mesenteric vessels. geons, and spine surgeons directed patient management with consensus on early spinal decompression and stabilization followed by interval debulking surgery and adjuvant therapy.

\section{Operation}

Percutaneous MI L-2 to ilium fixation with right to left over the top S-1 decompression was performed by the senior author (G.L.). A dual iliac screw $(7.5 \times 75 \mathrm{~mm}$ and $8 \times 95 \mathrm{~mm}$ ), dual rod $(5.5 \mathrm{~mm}$, cobalt chrome) construct was used for stabilizing the left lumbopelvic junction with minimal blood loss. For the right side, a dual screw-rod construct was planned as well; however, because significant rapid bleeding was encountered during keyhole decompression on the right $\mathrm{S}-1$ region, which accounted for approximately $600 \mathrm{ml}$ of blood loss, a decision was made to have only a single iliac screw $(9.0 \times 95 \mathrm{~mm})$ on the right side to reduce further tissue manipulation (Fig. 3). Total intraoperative blood loss was $800 \mathrm{ml}$.

\section{Postoperative Course}

The patient's postoperative course was uneventful with full recovery and symptom resolution. At the 1-year follow-up, there was minimal back and leg pain and no signs of screw prominence. However, the patient had declined abdominal tumor resection as well as adjuvant therapy and had been pursuing alternative treatment. Despite an increase in bone destruction, radiographs at the last 

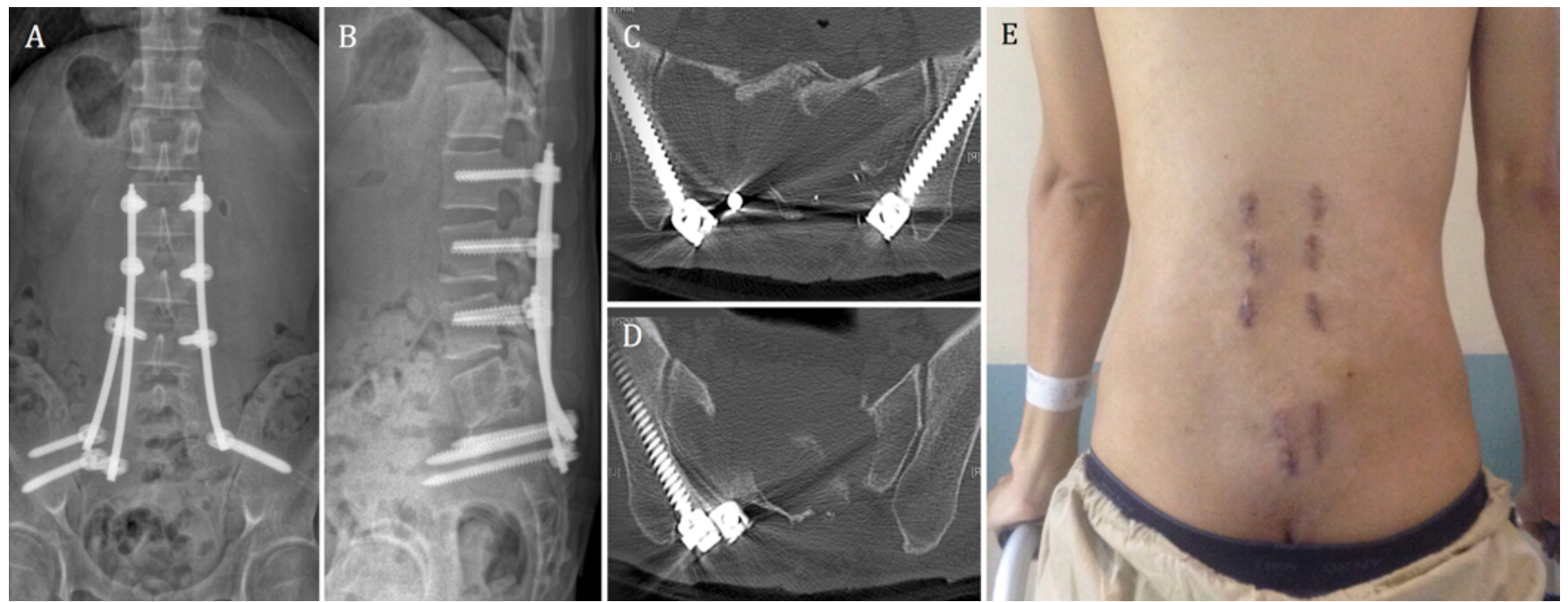

FIG. 3. An L-2 to ilium fixation was performed with a left dual iliac screw, dual rod construct (A-D). Wound review 1 week postsurgery (E). Figure is available in color online only.

follow-up did not show any evidence of construct failure (Fig. 4).

\section{Dual Iliac Screw, Dual Rod MI Surgical Technique}

A detailed description of an MI single iliac screw insertion technique was reported in our previous study. ${ }^{11} \mathrm{~A}$ modification of that technique for dual iliac screw, dual rod insertion is reported as follows. 1) The obturator outlet view $^{16}$ of the ilium guides the entry point for percutaneous iliac screw insertion. The teardrop view is created by rotating a fluoroscope approximately $45^{\circ}$ axial in line with the iliac wing and then tilting it $45^{\circ}$ caudally. 2) The inferior screw entry point is marked first by aiming toward the inferior aspect of the teardrop view. A 2-cm skin incision approximately $1 \mathrm{~cm}$ medial to the iliac crest and inferior
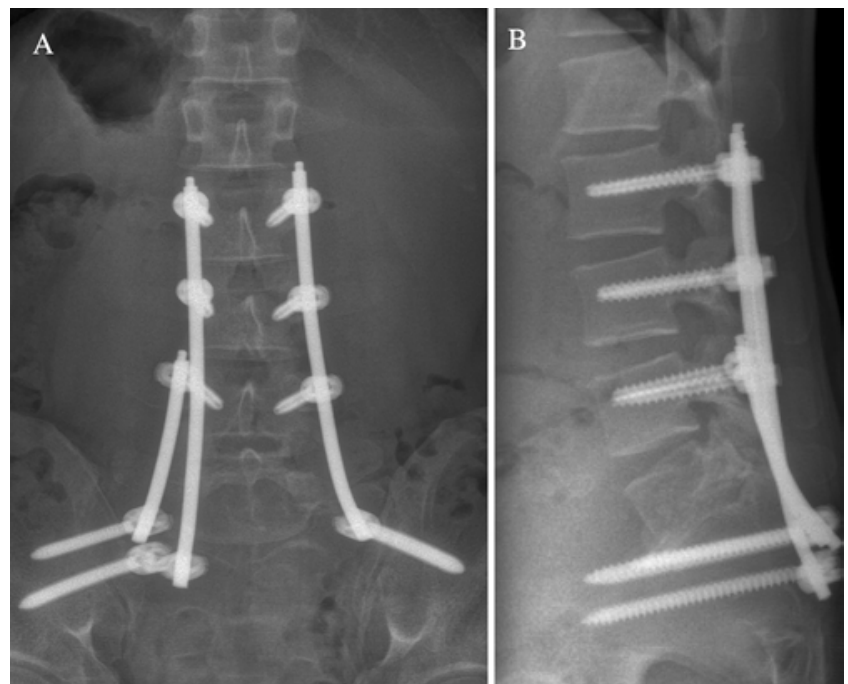

FIG. 4. Stable fixation at the 1-year follow-up despite increased bone destruction (left-right anteroposterior view, A; lateral view, B). to posterior superior iliac spine is made as the screw entry site. 3) Finger dissection is used to locate the medial iliac wall inferior to the iliac crest to guide screw entry, avoiding sacroiliac joint violation and screw head prominence above the iliac crest. 4) Guided by the radiological view of the teardrop region, laterally aiming at the anterior inferior iliac spine, the first Jamshidi needle is inserted in the medial wall, inferior to the iliac crest and above the sacroiliac joint (Fig. 5). 5) A second Jamshidi needle is then inserted $1-2 \mathrm{~cm}$ cranial and parallel to the inferior Jamshidi. This maps the trajectory of the superior screw. Guidewires are then passed through the Jamshidi needles, followed by length measurement, tapping, and screw insertions. 6) For a stable balanced construct, we recommend spinal instrumentation 3 levels cephalic to the unstable
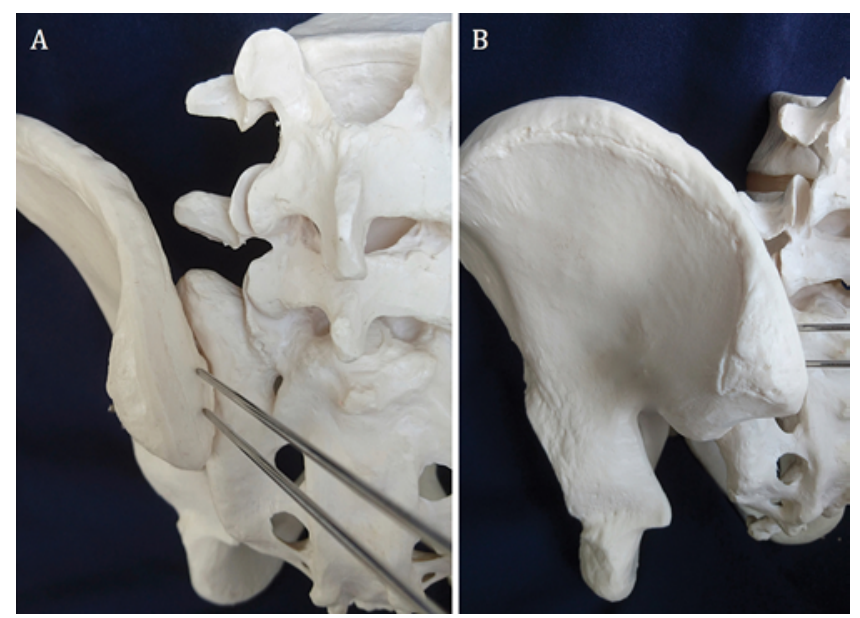

FIG. 5. Model showing needle entry points and trajectories. The first needle is inserted in the medial wall, inferior to the iliac crest and above the sacroiliac joint, followed by a second needle inserted $1-2 \mathrm{~cm}$ cranial and parallel to the first $(\mathbf{A})$. Both needles directed toward the anteroinferior iliac spine with passage above the sciatic notch $(B)$. Figure is available in color online only. 
metastatic pathology. 7) Careful pedicle screw placements are needed to ease dual rod placement. On the left side, a more laterally placed pedicle screw was inserted at L-4 immediately proximal to the pathology site. More medially placed screws were inserted at the adjacent cephalic sites (Fig. 3). 8) The first, long, percutaneously contoured rod was placed to engage the L-2 and L-3 pedicle screws to the more caudally placed iliac screw via a side connector. 9) The second, short, percutaneously contoured rod was directly placed lateral to the first rod from L-4 to the proximal iliac screw, without side connectors.

\section{Discussion}

The lumbosacral junction is an epicenter of biomechanical stress, with the sacrum being a major stabilizer and load-transfer medium from the axial to appendicular skeleton. Pathology of the LSJ disrupts this load transmission axis and mandates a strong spinal construct for its reconstitution. Unlike historical spinopelvic fixation techniques, iliac screw constructs offer a superior biomechanical profile in that they extend anterior to the lumbosacral pivot and create a long lever arm within the ilium to counteract the LSJ forces. ${ }^{12}$ In vitro biomechanical comparisons between various lumbopelvic fixation techniques have shown iliac screw fixation to be most resistant to stress failure. ${ }^{3,10}$ Clinical advantages of using single iliac screw fixation with a single rod have been well described in the adult spinal deformity and metastatic spine literature. , $, 11,17^{-17}$

In the presence of extensive LSJ metastasis, however, even iliac screw constructs can have biomechanical constraints. In cadaveric sacrectomy models, iliac screws were shown to restore only $60 \%$ of the stability of the initial condition..$^{21}$ In a biomechanical study, Yu et al. demonstrated the improved stability of dual over single iliac screws. ${ }^{19}$ In 2008 Fujibayashi et al. described a series of open dual iliac screw insertions in patients with metastatic LSJ disease. ${ }^{5}$ Acharya and colleagues also reported dual iliac screws for iliolumbar fixation in patients with extensive LSJ instability. ${ }^{1}$ Furthermore, dual iliac screws give surgeons the option of using dual rods for stronger spinopelvic fixation. Mindea et al. compared the biomechanical strength between single and dual screw-rod constructs in a cadaveric total sacrectomy model and found that the double screw-rod construct offered the greatest stability under stress loading. ${ }^{13}$ In a biomechanical study by Hallager et al., a pedicle subtraction osteotomy model demonstrated the greatest reduction in motion and rod strain with the use of accessory rods. ${ }^{6}$ In the current case, extensive sacral neoplastic disease, especially on the left side, would have reduced screw hold and heightened the risk of iliac screw failure. Hence, for added stability, a dual screw-rod construct was created on the left side.

Despite the biomechanical advantage of a dual iliac screw-rod construct, a conventional open surgical approach causes significant soft tissue destruction and blood loss. Metastases from paraganglionomas are hypervascular, and this characteristic would have further increased complication in the featured case. The use of an MI approach reduces these complications. Wang et al. reported the successful use of a percutaneous single iliac screwrod construct with minimal complications in degenerative lumbar spine deformity. ${ }^{18}$ Our group furthered MI single iliac screw fixation's role as a palliative option in a study of metastatic LSJ deformity and showed no construction failure more than 13 months postsurgery. ${ }^{11}$ In the current case report, we employed the same palliative approach; however, to meet the functional demands of this young patient with extensive metastatic LSJ destruction, we further modified our MI single iliac screw-rod technique to an MI double iliac screw, double rod construct technique to ensure stability. Essentially, our goal was symptom control along with short- and medium-term spinal stabilization through minimal soft tissue disruption. Hence, achieving spinal fusion was not a consideration in this palliative setting. Neither did we employ cross-linkages, as they would have required additional tissue dissection and manipulation.

One of the limitations to this study is the nature of this single case with its limited follow-up. However, at the 1-year review, even though the patient did not undergo retroperitoneal tumor load reduction or adjuvant therapy, the spinal construct had not failed and there was no recurrence of back pain or neurological deficits.

\section{Conclusions}

Percutaneous MI dual iliac screw fixation is a viable option in situations in which additional stability is required due to extensive neoplastic disease or young active individuals with increased functional demands. Shortterm results from this report are encouraging; however, more research is warranted to establish the procedure's long-term safety.

\section{References}

1. Acharya NK, Bijukachhe B, Kumar RJ, Menon VK: Iliolumbar fixation - the Amrita technique. J Spinal Disord Tech 21:493-499, 2008

2. Allen BL Jr, Ferguson RL: The Galveston technique for $\mathrm{L}$ rod instrumentation of the scoliotic spine. Spine (Phila Pa 1976) $7: 276-284,1982$

3. Cunningham BW, Sefter JC, Hu N, Kim SW, Bridwell KH, McAfee PC: Biomechanical comparison of iliac screws versus interbody femoral ring allograft on lumbosacral kinematics and sacral screw strain. Spine (Phila Pa 1976) 35:E198E205, 2010

4. Doita M, Harada T, Iguchi T, Sumi M, Sha H, Yoshiya S, et al: Total sacrectomy and reconstruction for sacral tumors. Spine (Phila Pa 1976) 28:E296-E301, 2003

5. Fujibayashi S, Neo M, Nakamura T: Palliative dual iliac screw fixation for lumbosacral metastasis. Technical note. J Neurosurg Spine 7:99-102, 2007

6. Hallager DW, Gehrchen M, Dahl B, Harris JA, Gudipally M, Jenkins S, et al: Use of supplemental short pre-contoured accessory rods and cobalt chrome alloy posterior rods reduces primary rod strain and range of motion across the pedicle subtraction osteotomy level: an in vitro biomechanical study. Spine (Phila Pa 1976) 41:E388-E395, 2016

7. Hyun SJ, Rhim SC, Kim YJ, Kim YB: A mid-term follow-up result of spinopelvic fixation using iliac screws for lumbosacral fusion. J Korean Neurosurg Soc 48:347-353, 2010

8. Kasten MD, Rao LA, Priest B: Long-term results of iliac wing fixation below extensive fusions in ambulatory adult 
patients with spinal disorders. J Spinal Disord Tech 23:e37-e42, 2010

9. Kostuik JP, Hall BB: Spinal fusions to the sacrum in adults with scoliosis. Spine (Phila Pa 1976) 8:489-500, 1983

10. Lebwohl NH, Cunningham BW, Dmitriev A, Shimamoto $\mathrm{N}$, Gooch L, Devlin V, et al: Biomechanical comparison of lumbosacral fixation techniques in a calf spine model. Spine (Phila Pa 1976) 27:2312-2320, 2002

11. Liu G, Hasan MY, Wong HK: Minimally invasive iliac screw fixation in treating painful metastatic lumbosacral deformity: a technique description and clinical results. Eur Spine J 25:4043-4051, 2016

12. McCord DH, Cunningham BW, Shono Y, Myers JJ, McAfee PC: Biomechanical analysis of lumbosacral fixation. Spine (Phila Pa 1976) 17 (8 Suppl):S235-S243, 1992

13. Mindea SA, Salehi SA, Ganju A, Rosner MK, O'Shaughnessy BA, Jorge A, et al: Lumbosacropelvic junction reconstruction resulting in early ambulation for patients with lumbosacral neoplasms or osteomyelitis. Neurosurg Focus 15(2):E6, 2003

14. Ohata N, Ozaki T, Kunisada T, Morimoto Y, Tanaka M, Inoue $\mathrm{H}$ : Extended total sacrectomy and reconstruction for sacral tumor. Spine (Phila Pa 1976) 29:E123-E126, 2004

15. Parenti G, Zampetti B, Rapizzi E, Ercolino T, Giachè V, Mannelli M: Updated and new perspectives on diagnosis, prognosis, and therapy of malignant pheochromocytoma/ paraganglioma. J Oncol 2012:872713, 2012

16. Schildhauer TA, McCulloch P, Chapman JR, Mann FA: Anatomic and radiographic considerations for placement of transiliac screws in lumbopelvic fixations. J Spinal Disord Tech 15:199-205, 2002

17. Tsuchiya K, Bridwell KH, Kuklo TR, Lenke LG, Baldus C: Minimum 5-year analysis of L5-S1 fusion using sacropelvic fixation (bilateral S1 and iliac screws) for spinal deformity. Spine (Phila Pa 1976) 31:303-308, 2006

18. Wang MY, Ludwig SC, Anderson DG, Mummaneni PV: Per- cutaneous iliac screw placement: description of a new minimally invasive technique. Neurosurg Focus 25(2):E17, 2008

19. Yu BS, Zhuang XM, Zheng ZM, Li ZM, Wang TP, Lu WW: Biomechanical advantages of dual over single iliac screws in lumbo-iliac fixation construct. Eur Spine J 19:1121-1128, 2010

20. Zhang HY, Thongtrangan I, Balabhadra RS, Murovic JA, Kim DH: Surgical techniques for total sacrectomy and spinopelvic reconstruction. Neurosurg Focus 15(2):E5, 2003

21. Zheng ZM, Yu BS, Chen H, Aladin DM, Zhang KB, Zhang JF, et al: Effect of iliac screw insertion depth on the stability and strength of lumbo-iliac fixation constructs: an anatomical and biomechanical study. Spine (Phila Pa 1976) 34:E565-E572, 2009

\section{Disclosures}

In 2016, National University Health System provided two spine courses in partnership with DePuy Spine and K2M.

\section{Author Contributions}

Conception and design: Liu. Acquisition of data: both authors. Analysis and interpretation of data: both authors. Drafting the article: both authors. Critically revising the article: both authors. Reviewed submitted version of manuscript: both authors. Approved the final version of the manuscript on behalf of both authors: Liu.

\section{Correspondence}

Gabriel Liu, University Orthopaedic, Hand and Reconstructive Surgery Cluster, National University Health System, 1E Kent Ridge Rd., NUHS Tower Block, Level 11, Singapore 11922, Singapore. email: gabriel_liu@nuhs.edu.sg. 